\title{
A STOCHASTIC PRÉKOPA-LEINDLER INEQUALITY FOR LOG-CONCAVE FUNCTIONS
}

\author{
PETER PIVOVAROV \& JESÚS REBOLLO BUENO
}

\begin{abstract}
The Brunn-Minkowski and Prékopa-Leindler inequalities admit a variety of proofs that are inspired by convexity. Nevertheless, the former holds for compact sets and the latter for integrable functions so it seems that convexity has no special signficance. On the other hand, it was recently shown that the Brunn-Minkowski inequality, specialized to convex sets, follows from a local stochastic dominance for naturally associated random polytopes. We show that for the subclass of log-concave functions and associated stochastic approximations, a similar stochastic dominance underlies the Prékopa-Leindler inequality.
\end{abstract}

\section{INTRODUCTION}

The Brunn-Minkowski inequality governs the behavior of volume and Minkowski addition of compacts sets $K, L \subseteq \mathbb{R}^{n}$ :

$$
|K+L|^{1 / n} \geq|K|^{1 / n}+|L|^{1 / n}
$$

see [45, 23. As an isoperimetric principle, (1.1) can equivalently be stated in the form

$$
|K+L| \geq\left|K^{*}+L^{*}\right|,
$$

where $A^{*}$ denotes the Euclidean ball of the same volume as $A$. In 38, it is shown that when one specializes to convex bodies $K, L \subseteq \mathbb{R}^{n}$, then (1.2) admits a stronger stochastic formulation for random polytopes. Namely, for each convex body $K \subseteq \mathbb{R}^{n}$, we sample independent random vectors $X_{1}, \ldots, X_{N}$ uniformly in $K$ and associate a random polytope

$$
[K]_{N}=\operatorname{conv}\left\{X_{1}, \ldots, X_{N}\right\}
$$

where conv means convex hull. Then sampling independent random vectors in each of the bodies in (1.2) (on a common underlying probability space $(\Omega, \mathcal{A}, \mathbb{P}))$ leads to the following for each $\alpha \geq 0$,

$$
\mathbb{P}\left(\left|[K]_{N}+[L]_{M}\right|>\alpha\right) \geq \mathbb{P}\left(\left|\left[K^{*}\right]_{N}+\left[L^{*}\right]_{M}\right|>\alpha\right) .
$$

By the law of large numbers, as $N, M \rightarrow \infty$, the random polytopes converge to their ambient bodies. Thus (1.2) follows from a "local" stochastic 
dominance for random polytopes that naturally approximate convex bodies. Inequalities for the expected volume of the polytopes $[K]_{N}$ have a long history in stochastic geometry, including Blaschke's resolution of Sylvester's four point problem [6], and its generalizations to higher dimensions by Busemann [13] and Groemer [27]. We explicitly mention only Groemer's inequality on random polytopes which is recovered from (1.3) when working with one body $K$ :

$$
\mathbb{E}\left|[K]_{N}\right| \geq \mathbb{E}\left|\left[K^{*}\right]_{N}\right|
$$

For related work, see, e.g., [15, 26, 37, 38], 45, Chapter 10] and the references therein.

The Prékopa-Leindler inequality [31, 40, 41] asserts that for integrable functions $f, g, h: \mathbb{R}^{n} \rightarrow[0, \infty)$ and $0<\lambda<1$, if

$$
h(\lambda x+(1-\lambda) y) \geq f(x)^{\lambda} g(y)^{1-\lambda}
$$

for all $x, y \in \mathbb{R}^{n}$, then

$$
\int h \geq\left(\int f\right)^{\lambda}\left(\int g\right)^{1-\lambda}
$$

By taking $f=\mathbb{1}_{K}, g=\mathbb{1}_{L}$, and $h=\mathbb{1}_{\lambda K+(1-\lambda) L}$ (1.6) implies (1.1). In this sense the Prékopa-Leindler inequality can be viewed as a functional extension of the Brunn-Minkowski inequality. Related variations of (1.6) provide a basis for functional versions of Brunn's principle; see Borell [8], Rinott [42, Brascamp and Lieb [9, 10]. The reach of the Prékopa-Leindler inequality now extends into various branches of analysis, geometry, probability, information theory, among other fields; see, e.g., [23, 17, 18, 19, 20, 34] and the references therein.

As for the Brunn-Minkowski inequality, our focus here is on a form of (1.6) involving symmetric decreasing rearrangements $f^{*}$ and $g^{*}$ (see $\$ 2$ for definitions). Brascamp and Lieb proved (1.6) via rearrangement inequalities through the reverse Young inequality [9]. More recently, Melbourne [35] derived families of rearrangement inequalities refining (1.6); the version we study here involves Borel measurable $f, g: \mathbb{R}^{n} \rightarrow[0, \infty), 0<\lambda<1$ and the sup-convolution

$$
\left(f \star_{\lambda} g\right)(v)=\sup \left\{f^{\lambda}(x) g^{1-\lambda}(y): v=\lambda x+(1-\lambda) y\right\},
$$

for which one has

$$
\int_{\mathbb{R}^{n}}\left(f \star_{\lambda} g\right)(v) d v \geq \int_{\mathbb{R}^{n}}\left(f^{*} \star_{\lambda} g^{*}\right)(v) d v .
$$


We will show that a similar "local" stochastic dominance underlies (1.7) when one focuses only on log-concave functions $f$ and $g$, i.e., when $\log f$ and $\log g$ are concave on their supports.

To define our stochastic model, for each integrable log-concave function $f$ : $\mathbb{R}^{n} \rightarrow[0, \infty)$, we sample independent random vectors $\left(X_{1}, Z_{1}\right), \ldots,\left(X_{N}, Z_{N}\right)$ in $\mathbb{R}^{n} \times[0, \infty)$ according to the uniform Lebesgue measure on the region under the graph of $f$ :

$$
G_{f}:=\left\{(x, z) \in \mathbb{R}^{n} \times[0, \infty): x \in \operatorname{supp} f, z \leq f(x)\right\} .
$$

We denote by $[f]_{N}$ the least log-concave function supported on the set $\operatorname{conv}\left\{X_{1}, \ldots, X_{N}\right\}$ with $[f]_{N}\left(X_{i}\right) \geq Z_{i}, i=1, \ldots, N$. In other words, for the convex domain

$$
H_{f, N}:=\operatorname{conv}\left\{\left(X_{1}, \log Z_{1}\right), \ldots,\left(X_{N}, \log Z_{N}\right)\right\},
$$

we set

$$
[f]_{N}(x):=\exp \left(\sup \left\{z:(x, z) \in H_{f, N}\right\}\right) .
$$

This model of approximation works equally well for $f^{*}$. Indeed, when $f$ is log-concave, the same is true of $f^{*}$. Thus for independent random samples for each of the log-concave functions $f, g, f^{*}, g^{*}$, we can now state our main result.

Theorem 1.1. Let $f, g: \mathbb{R}^{n} \rightarrow[0, \infty)$ be log-concave functions and $N, M>$ $n+1$. Then, for any $\alpha>0$,

$$
\mathbb{P}\left(\int_{\mathbb{R}^{n}}\left([f]_{N} \star_{\lambda}[g]_{M}\right)(v) d v>\alpha\right) \geq \mathbb{P}\left(\int_{\mathbb{R}^{n}}\left(\left[f^{*}\right]_{N} \star_{\lambda}\left[g^{*}\right]_{M}\right)(v) d v>\alpha\right) .
$$

When $N, M \rightarrow \infty$, the latter implies (1.7). Thus, in this sense, a "local" stochastic dominance underlies the Prékopa-Leindler inequality for logconcave $f$ and $g$.

There is a solid foundation for extending geometric inequalities from convex sets to log-concave functions or more general classes, e.g., [4, 29, 2, 21, 30, 36, 17, 1. While a number of isoperimetric inequalities in addition to (1.1) have found stochastic versions [38, no similar progress has been made for stochastic functional inequalities. Theorem 1.1 is a first step towards stochastic isoperimetric inequalities for random functions. Working with just one function $f$, we obtain a functional analogue of Groemer's result (1.4) for random polytopes.

Theorem 1.2. Let $f: \mathbb{R}^{n} \rightarrow[0, \infty)$ be an integrable log-concave function and $N>n+1$. Then, for any $\alpha>0$,

$$
\mathbb{P}\left(\int_{\mathbb{R}^{n}}[f]_{N}(x) d x>\alpha\right) \geq \mathbb{P}\left(\int_{\mathbb{R}^{n}}\left[f^{*}\right]_{N}(x) d x>\alpha\right) .
$$


The techniques from [38] leading to the stochastic Brunn-Minkowski inequality (1.3) involve random convex sets. New ingredients are needed to treat functions and their stochastic approximations. Various proofs of the Prékopa-Leindler (1.6) inequality rely on the Brunn-Minkowski inequality. Of particular significance to our approach is the work of Klartag [28]; he derives various functional inequalities, including (1.6), as "marginals" of geometric inequalities for convex bodies in higher dimensions. The reduction to sets in higher dimensions fits well with the stochastic approximation that we use. In particular, they interface well with multiple integral rearrangement inequalities of Rogers [43], Brascamp-Lieb-Luttinger [11] and Christ [16].

\section{Preliminaries}

Let $K$ be a convex set in $\mathbb{R}^{n}, \theta$ on the unit sphere $\mathbb{S}^{n-1}$ and $P:=P_{\theta^{\perp}}$ the orthogonal projection onto $\theta^{\perp}$. We define $u_{K}: P K \rightarrow \mathbb{R}$ by

$$
u_{K}(y):=u(K, y):=\sup \{\lambda: y+\lambda \theta \in K\}
$$

and $\ell_{K}: P K \rightarrow \mathbb{R}$ by

$$
\ell_{K}(y):=\ell(K, y):=\inf \{\lambda: y+\lambda \theta \in K\} .
$$

Notice that, for $K$ convex, $u_{K}$ and $\ell_{K}$ are concave and convex, respectively.

We recall that the Steiner symmetral of a non-empty compact set $A \subseteq \mathbb{R}^{n}$ with respect to $\theta^{\perp}, S_{\theta^{\perp}} A$, is the set with the property that for each line $l$ orthogonal to $\theta^{\perp}$ and meeting $A$, the set $l \cap S_{\theta^{\perp}} A$ is a closed segment with midpoint on $\theta^{\perp}$ and length equal to that of the set $l \cap A$. The mapping $S_{\theta^{\perp}}: A \rightarrow S_{\theta^{\perp}} A$ is called the Steiner symmetrization of $A$ with respect to $\theta^{\perp}$. In particular, if $K$ is a convex body

$$
S_{\theta^{\perp}} K=\left\{x+\lambda \theta: x \in P K,-\frac{u_{K}(x)-\ell_{K}(x)}{2} \leq \lambda \leq \frac{u_{K}(x)-\ell_{K}(x)}{2}\right\} .
$$

This shows that $S_{\theta^{\perp}} K$ is convex, since the function $u_{K}-\ell_{K}$ is concave. Moreover, $S_{\theta^{\perp}} K$ is symmetric with respect to $\theta^{\perp}$, it is closed, and by Fubini's theorem it has the same volume as $K$.

Let $A \subseteq \mathbb{R}^{n}$ be a Borel set with finite Lebesgue measure. The symmetric rearrangement, $A^{*}$, of $A$ is the open ball with center at the origin whose volume is equal to the measure of $A$. Since we choose $A^{*}$ to be open, $\mathbb{1}_{A^{*}}$ is lower semicontinuous. The symmetric decreasing rearrangement of $\mathbb{1}_{A}$ is defined by $\mathbb{1}_{A}^{*}=\mathbb{1}_{A^{*}}$. We say a Borel measurable function $f: \mathbb{R}^{n} \rightarrow[0, \infty)$ vanishes at infinity if for every $t>0$, the set $\left\{x \in \mathbb{R}^{n}: f(x)>t\right\}$ has finite Lebesgue measure. In such a case, the symmetric decreasing rearrangement 
$f^{*}$ is defined by

$$
f^{*}(x)=\int_{0}^{\infty} \mathbb{1}_{\{f>t\}}^{*}(x) d t=\int_{0}^{\infty} \mathbb{1}_{\{f>t\}^{*}}(x) d t .
$$

Observe that $f^{*}$ is radially symmetric, radially decreasing, and equimeasurable with $f$, i.e., $\{f>t\}$ and $\left\{f^{*}>t\right\}$ have the same measure for each $t>0$. Let $\left\{e_{1}, \ldots, e_{n}\right\}$ be an orthonormal basis of $\mathbb{R}^{n}$ such that $e_{1}=\theta$. Then, for $f$ vanishing at infinity, the Steiner symmetral $f(\cdot \mid \theta)$ of $f$ with respect to $\theta^{\perp}$ is defined as follows: set $f_{\left(x_{2}, \ldots, x_{n}\right), \theta}(t)=f\left(t, x_{2}, \ldots, x_{n}\right)$ and define $f^{*}\left(t, x_{2}, \ldots, x_{n} \mid \theta\right):=\left(f_{\left(x_{2}, \ldots, x_{n}\right), \theta}\right)^{*}(t)$. In other words, we obtain $f^{*}(\cdot \mid \theta)$ by rearranging $f$ along every line parallel to $\theta$. We refer to the books [32, 46] or the introductory notes [12] for further background material on rearrangement of functions.

\section{Approximation of log-Concave functions}

We start by recalling an approach to derving integral inequalities for functions by using certain higher-dimensional bodies of revolution. This method was used by Artstein, Klartag and Milman in [2] to extend Ball's functional Blaschke-Santaló inequality [4]; see [22, 5] for further developments. Such bodies were also used in the first derivation of the functional affine isoperimetric inequality [3]; see also [14]. As we mentioned in the introduction, Klartag [28] used the method to prove the Prékopa-Leindler inequality and we will review several key points for later use.

A function $f: \mathbb{R}^{n} \rightarrow[0, \infty)$ is called log-concave if $\log f$ is concave on its support. In accordance with the usage of $s$-concavity in [2, 28], we say $f$ is $s$-concave if $f^{1 / s}$ is concave on its support; this is not the same as other common uses of the term, e.g. [8], however, it fits with the approach taken here. In particular, any $s$-concave function, for $s>0$, is also log-concave. A useful approximation of a log-concave function $f$ by $s$-concave functions $f_{s}$ is given by

$$
f_{s}(x)=\left(1+\frac{\log f(x)}{s}\right)_{+}^{s},
$$

where $x_{+}=\max \{x, 0\}$. With this choice, $f_{s} \leq f$ for all $s>0$, and since a log-concave function is continuous on its support one has $f_{s} \rightarrow f$ locally uniformly on $\mathbb{R}^{n}$ as $s \rightarrow \infty$.

Let $\left(e_{1}, \ldots, e_{n+s}\right)$ be an orthonormal basis of $\mathbb{R}^{n+s}=\mathbb{R}^{n} \times \mathbb{R}^{s}$. For a measurable function $f: \mathbb{R}^{n} \rightarrow[0, \infty)$, we can associate the set

$$
\mathcal{K}_{f}^{s}:=\left\{(x, y) \in \mathbb{R}^{n} \times \mathbb{R}^{s}: x \in \overline{\operatorname{supp} f},|y| \leq f^{1 / s}(x)\right\},
$$


where $|\cdot|$ denotes the usual Euclidean norm. With this terminology, Brunn's principle means that a function on $\mathbb{R}^{n}$ is $s$-concave if and only if it is a marginal of a uniform measure on a convex body in $\mathbb{R}^{n+s}$. Thus $\mathcal{K}_{f}^{s}$ is convex if and only if $f$ is $s$-concave. Moreover,

$$
f(x)=\kappa_{s}^{-1} \int_{\mathbb{R}^{s}} \mathbb{1}_{\mathcal{K}_{f}^{s}}(x, y) d y,
$$

where $\kappa_{s}$ is the volume of the $s$-dimensional Euclidean ball and hence

$$
\int_{\mathbb{R}^{n}} f(x) d x=\kappa_{s}^{-1}\left|\mathcal{K}_{f}^{s}\right|
$$

We also recall the notion of homothety of bodies, the $s$-Minkowski sum of two non-negative functions on $\mathbb{R}^{n}$, and their relation with the set (3.2). Let $\lambda>0$. We define the function $\lambda \cdot_{s} f: \mathbb{R}^{n} \rightarrow[0, \infty)$ to be

$$
\left[\lambda \cdot{ }_{s} f\right](x)=\lambda^{s} f\left(\frac{x}{\lambda}\right) .
$$

This way $\mathcal{K}_{\lambda \cdot s f}^{s}=\lambda \mathcal{K}_{f}^{s}$ so $\lambda \cdot_{s} f$ is a functional analog of homothety of bodies, where if $f$ is an $s$-concave function, so is $\lambda \cdot s f$. Define also the $s$-Minkowski sum of two functions $f, g: \mathbb{R}^{n} \rightarrow[0, \infty)$ as

$\left[f \oplus_{s} g\right](v)=\sup \left\{\left(f(x)^{\frac{1}{s}}+g(y)^{\frac{1}{s}}\right)^{s}: v=x+y, x \in \operatorname{supp}(f), y \in \operatorname{supp}(g)\right\}$

whenever $v \in \operatorname{supp}(f)+\operatorname{supp}(g)$. If not, we set $\left[f \oplus_{s} g\right](v)=0$. This function is $s$-concave whenever $f$ and $g$ are, and

$$
\mathcal{K}_{f \oplus s}^{s}=\mathcal{K}_{f}^{s}+\mathcal{K}_{g}^{s} .
$$

By (3.2) the latter body is convex when $f$ and $g$ are $s$-concave. Let us also denote

$$
\left(f \star_{\lambda, s} g\right)(v):=\left((\lambda \cdot s f) \oplus_{s}((1-\lambda) \cdot s g)\right)(v),
$$

and, as in the introduction,

$$
\left(f \star_{\lambda} g\right)(v):=\sup \left\{f^{\lambda}(x) g^{1-\lambda}(y): v=\lambda x+(1-\lambda) y\right\} .
$$

Therefore it follows that

$$
\mathcal{K}_{f \star \lambda, s}^{s}=\mathcal{K}_{\lambda \cdot s}^{s}+\mathcal{K}_{(1-\lambda) \cdot s}^{s}=\mathcal{K}_{f}^{s}+_{\lambda} \mathcal{K}_{g}^{s},
$$

where we have defined

$$
K+{ }_{\lambda} L:=\lambda K+(1-\lambda) L .
$$

In this way, the Prekopa-Leindler inequality is derived in [28] as a "marginal" of the Brunn-Minkowski inequality in $\mathbb{R}^{n+s}$ when $s \rightarrow \infty$. We cannot directly apply the stochastic Brunn-Minkowski inequality (1.3) to the bodies $K=\mathcal{K}_{f}^{s}$ and $L=\mathcal{K}_{g}^{s}$ as this would involve different measures in each 
dimension $n+s$ and an increasing number of samples in each body. Instead, we revisit the proof of (1.3) and study operations beyond the convex hull of points that can be used in our stochastic approach. The following lemma provides a needed link. Henceforth, we denote the $s$-dimensional Euclidean ball centered at a point $x \in \mathbb{R}^{n}$ and $\rho \geq 0$ by

$$
B_{\rho}^{s}(x)=\left\{(x, \widehat{z}) \in \mathbb{R}^{n} \times \mathbb{R}^{s}:|\widehat{z}| \leq \rho\right\} .
$$

Lemma 3.1. Let $w_{i}=\left(x_{i}, z_{i}\right) \in \mathbb{R}^{n} \times[0, \infty)$ for $i=1, \ldots, N$ and $s \in \mathbb{N}$. Let $T=T_{\left\{w_{i}\right\}, s}$ be the least s-concave function supported on $\operatorname{conv}\left\{x_{1}, \ldots, x_{N}\right\}$ such that $T\left(x_{i}\right) \geq z_{i}, i=1, \ldots, N$, i.e.,

$$
T(x)=\sup \left\{z^{s} \in \mathbb{R}:(x, z) \in \operatorname{conv}\left\{\left(x_{i}, r_{i}\right)\right\}_{i=1}^{N}\right\},
$$

where $r_{i}=z_{i}^{1 / s}, i=1, \ldots, N$. Then

$$
\mathcal{K}_{T}^{s}=\operatorname{conv}\left\{B_{r_{1}}^{s}\left(x_{1}\right), \ldots, B_{r_{N}}^{s}\left(x_{N}\right)\right\} .
$$

Proof. For $x \in \operatorname{conv}\left\{x_{1}, \ldots, x_{N}\right\}$ and $\widehat{z} \in \mathbb{R}^{s}$ with $(x, \widehat{z}) \in \mathcal{K}_{T}^{s}$, there exists non-negative $c_{1}, \ldots, c_{N}$ with $\sum_{i} c_{i}=1$ such that

$$
|\widehat{z}| \leq T(x)^{1 / s}=\sum_{i=1}^{N} c_{i} r_{i},
$$

and $x=\sum_{i=1}^{N} c_{i} x_{i}$. Thus, denoting $r:=\sum_{i=1}^{N} c_{i} r_{i}$, we have

$$
(x, \widehat{z}) \in B_{r}^{s}(x) \subseteq \operatorname{conv}\left\{B_{r_{1}}^{s}\left(x_{1}\right), \ldots, B_{r_{N}}^{s}\left(x_{N}\right)\right\} .
$$

On the other hand, let $(x, \widehat{z}) \in \operatorname{conv}\left\{B_{r_{1}}^{s}\left(x_{1}\right), \ldots, B_{r_{N}}^{s}\left(x_{N}\right)\right\}$ so that for some $\left(c_{i}\right)$ with $c_{i} \geq 0, \sum_{i} c_{i}=1,(x, \widehat{z})=\sum_{i=1}^{N} c_{i}\left(x_{i}, \widehat{z}_{i}\right)$ where $\widehat{z}_{i} \in \mathbb{R}^{s}$ and $\left|\widehat{z}_{i}\right| \leq r_{i}$, for $i=1, \ldots, N$. Therefore,

$$
|\widehat{z}| \leq \sum_{i=1}^{N} c_{i} r_{i} \leq T(x)^{1 / s},
$$

so $(x, \widehat{z}) \in \mathcal{K}_{T}^{s}$ as desired.

\section{Rearrangements and Steiner Convexity}

When an isoperimetric principle admits a proof by symmetrization, like (1.2) for example, it is often meaningful to instead carry out such symmetrization on a suitable (product) probability space. In [38, a variety of isoperimetric inequalities for convex sets are shown to admit stronger stochastic forms. A key tool in this approach involves multiple integral rearrangement inequalities of Rogers [43], and Brascamp-Lieb-Luttinger [1]. Christ's version [16] of the latter is especially well-suited for stochastic forms 
of isoperimetric inequalities; as in [38], the following formulation is convenient for our purpose.

Theorem 4.1. Let $f_{1}, \ldots, f_{N}$ be non-negative integrable functions on $\mathbb{R}^{n}$ and $F:\left(\mathbb{R}^{n}\right)^{N} \rightarrow[0, \infty)$. Then

$$
\begin{aligned}
& \int_{\left(\mathbb{R}^{n}\right)^{N}} F\left(x_{1}, \ldots, x_{N}\right) \prod_{i=1}^{N} f_{i}\left(x_{i}\right) d x_{1} \ldots d x_{N} \\
& \geq \int_{\left(\mathbb{R}^{n}\right)^{N}} F\left(x_{1}, \ldots, x_{N}\right) \prod_{i=1}^{N} f_{i}^{*}\left(x_{i}\right) d x_{1} \ldots d x_{N},
\end{aligned}
$$

whenever $F$ satisfies the following condition: for each $\theta \in \mathbb{S}^{n-1}$ and all $Y:=\left\{y_{1}, \ldots, y_{N}\right\} \subseteq \theta^{\perp}$, the function $F_{Y}: \mathbb{R}^{N} \rightarrow[0, \infty)$ defined by

$$
F_{Y, \theta}\left(t_{1}, \ldots, t_{N}\right):=F\left(y_{1}+t_{1} \theta, \ldots, y_{N}+t_{N} \theta\right)
$$

is even and quasi-convex.

The condition on $F$ allows the theorem to be proved via iterated Steiner symmetrization; as in [38, we will call such functions $F$ Steiner convex (which differs from the terminology in [16]). This condition interfaces well with generalizations of Steiner symmetrization like shadow systems, e.g., [44, 15]; see [38] for further background and references. For context, we recall only several examples before treating the functionals involved in our main theorems.

A fundamental example of a Steiner convex function is the absolute value of the determinant, $F\left(x_{1}, \ldots, x_{n}\right)=\left|\operatorname{det}\left(\left[x_{1}, \ldots, x_{n}\right]\right)\right|($ when $N=n)$; this is the key property behind Busemann's random simplex inequality [13]. More generally, Groemer [27] showed that for $N>n$, the functional

$$
F\left(x_{1}, \ldots, x_{N}\right)=\left|\operatorname{conv}\left\{x_{1}, \ldots, x_{N}\right\}\right|
$$

is also Steiner convex. While the latter examples involve points $x_{i}$, analogous results hold for convex hulls of Euclidean balls

$$
B_{\rho_{i}}\left(x_{i}\right):=\left\{u \in \mathbb{R}^{n}:\left|u-x_{i}\right| \leq \rho_{i}\right\} .
$$

Indeed, in [39], Pfiefer showed that for $\rho_{1}, \ldots, \rho_{N} \geq 0$, the functional

$$
F\left(x_{1}, \ldots, x_{N}\right)=\left|\operatorname{conv}\left\{B_{\rho_{1}}\left(x_{1}\right), \ldots, B_{\rho_{N}}\left(x_{N}\right)\right\}\right|
$$

satisfies the Steiner convexity property.

In [37, the functional in (4.1) was generalzed to include operations beyond the convex hull. Namely, let $C \subseteq \mathbb{R}^{N}$ be a compact convex set; for 
$x_{1}, \ldots, x_{N}$, we view the $n \times N$ matrix $\left[x_{1}, \ldots, x_{N}\right]$ as an operator from $\mathbb{R}^{N}$ to $\mathbb{R}^{n}$. Then

$$
\left[x_{1}, \ldots, x_{N}\right] C=\left\{\sum_{i} c_{i} x_{i}: c=\left(c_{i}\right) \in C\right\}
$$

and

$$
F\left(x_{1}, \ldots, x_{N}\right)=\left|\left[x_{1}, \ldots, x_{N}\right] C\right|
$$

is Steiner convex [37]. In particular, the functionals in the stochastic BrunnMinkowski inequality (1.3) and Groemer's inequality (1.4) fit naturally in this framework. Indeed, for independent random vectors $X_{1} \ldots, X_{N}$ in $K$, and $X_{N+1}, \ldots, X_{N+M}$ in $L$, we have

$$
[K]_{N}=\left[X_{1}, \ldots, X_{N}\right] C_{N}
$$

and

$$
\begin{aligned}
{[K]_{N}+[L]_{M} } & =\left[X_{1}, \ldots, X_{N}\right] C_{N}+\left[X_{N+1}, \ldots, X_{N+M}\right] C_{M} \\
& =\left[X_{1}, \ldots, X_{N+M}\right]\left(C_{N}+\widehat{C}_{M}\right),
\end{aligned}
$$

where $C_{k}=\operatorname{conv}\left\{e_{1}, \ldots, e_{k}\right\}$ for $k=N, M$ and $\widehat{C}_{M}=\operatorname{conv}\left\{e_{N+1}, \ldots, e_{N+M}\right\}$.

As noted in [38], convex operations on points in (4.3) can be combined with Euclidean balls (as in (4.2) ) by using the notion of $\mathcal{M}$-addition. The latter operation was studied in depth by Gardner, Hug and Weil in [25] as a unifying framework for operations in $L_{p}$ and Orlicz Brunn-Minkowski theory; see, e.g., [24, 33. for further references and background. For $\mathcal{M} \subseteq \mathbb{R}^{N}$ and subsets $K_{1}, \ldots, K_{N}$ in $\mathbb{R}^{n}$, their $\mathcal{M}$-combination is defined by

$$
\oplus_{\mathcal{M}}\left(K_{1}, \ldots, K_{N}\right)=\left\{\sum_{i=1}^{N} m_{i} x_{i}: x_{i} \in K_{i},\left(m_{1}, \ldots, m_{n}\right) \in \mathcal{M}\right\} .
$$

When $K_{1}, \ldots, K_{N}$ are convex and $\mathcal{M}$ is convex and contained in the positive orthant, then $\oplus_{\mathcal{M}}\left(K_{1}, \ldots, K_{N}\right)$ is convex [25, Theorem 6.1]. With this notation, for $C=\mathcal{M}$,

$$
\oplus_{C}\left(B_{\rho_{1}}\left(x_{1}\right), \ldots, B_{\rho_{N}}\left(x_{N}\right)\right)=\left\{\sum_{i=1}^{N} c_{i} u_{i}: u_{i} \in B_{\rho_{i}}\left(x_{i}\right), c=\left(c_{i}\right) \in C\right\} .
$$

To connect with the bodies of revolution $\mathcal{K}_{f}^{s} \subseteq \mathbb{R}^{n} \times \mathbb{R}^{s}$ defined in 93 , we use $\mathcal{M}$-combinations of $s$-dimenisonal Euclidean balls lying orthogonal to $\mathbb{R}^{n}$.

Proposition 4.2. Let $\rho_{1}, \ldots, \rho_{N} \in[0, \infty)$ and $C$ a convex set contained in the positive orthant. Then the function $F:\left(\mathbb{R}^{n}\right)^{N} \rightarrow[0, \infty)$ defined by

$$
F\left(x_{1}, \ldots, x_{N}\right)=\left|\oplus_{C}\left(B_{\rho_{1}}^{s}\left(x_{1}\right), \ldots, B_{\rho_{N}}^{s}\left(x_{N}\right)\right)\right|
$$

is Steiner convex. 
Proof. We suppose without loss of generality that $\theta=e_{1}$, where $\left\{e_{1}, \ldots, e_{n}\right\}$ denotes the standard basis in $\mathbb{R}^{n}$. Let $Y=\left\{y_{1}, \ldots, y_{N}\right\} \subseteq \theta^{\perp}$. We define the function $F_{Y}: \mathbb{R}^{N} \rightarrow[0, \infty)$ by

$$
\begin{aligned}
F_{Y}(t) & =\mid \oplus_{C}\left(B_{\rho_{1}}^{s}\left(\left(t_{1}, y_{1}\right)\right), \ldots, B_{\rho_{N}}^{s}\left(\left(t_{N}, y_{N}\right)\right) \mid\right. \\
& =\left|\left\{\sum_{i=1}^{N} c_{i}\left(t_{i}, y_{i}, \widehat{z}_{i}\right):\left|\widehat{z}_{i}\right| \leq \rho_{i}\right\}\right| .
\end{aligned}
$$

To check that $F_{Y}$ is even we note that the sets involved in the expressions for $F_{Y}(t)$ and $F_{Y}(-t)$ are reflections of each other about $\theta^{\perp}$ and hence have equal volume.

To prove convexity, let $r, t \in \mathbb{R}^{N}$. For $a=\left(a_{i}\right)$ in $\left\{r, t, \frac{r}{2}+\frac{t}{2}\right\}$, we write

$$
\oplus_{C}\left(\left\{B_{\rho_{i}}^{s}\left(a_{i}, y_{i}\right)\right\}\right):=\oplus_{C}\left(B_{\rho_{1}}^{s}\left(\left(a_{1}, y_{1}\right)\right), \ldots, B_{\rho_{N}}^{s}\left(\left(a_{N}, y_{N}\right)\right)\right) .
$$

Let $P: \mathbb{R}^{n+s} \rightarrow \theta^{\perp} \times \mathbb{R}^{s}$ denote the orthogonal projection. Note that

$$
D:=P\left(\oplus_{C}\left(\left\{B_{\rho_{i}}^{s}\left(a_{i}, y_{i}\right)\right\}\right)\right)
$$

is independent of $a$. Thus we define as in $42, u_{a}, \ell_{a}: D \rightarrow \mathbb{R}$ by

$$
u_{a}(v)=u\left(\oplus_{C}\left(\left\{B_{\rho_{i}}^{s}\left(a_{i}, y_{i}\right)\right\}\right), v\right)
$$

and

$$
\ell_{a}(v)=\ell\left(\oplus_{C}\left(\left\{B_{\rho_{i}}^{s}\left(a_{i}, y_{i}\right)\right\}\right), v\right) .
$$

Next, we set $u=\frac{1}{2}\left(u_{r}+u_{t}\right)$ and $\ell=\frac{1}{2}\left(\ell_{r}+\ell_{t}\right)$ and define

$$
E:=\{(\lambda, v): v \in D, \ell(v) \leq \lambda \leq u(v)\} .
$$

We claim that

$$
\oplus_{C}\left(\left\{B_{\rho_{i}}^{s}\left(\frac{r_{i}+t_{i}}{2}, y_{i}\right)\right\}\right) \subseteq E .
$$

To see this, let $w \in \oplus_{C}\left(\left\{B_{\rho_{i}}^{s}\left(\frac{r_{i}+t_{i}}{2}, y_{i}\right)\right\}\right)$ so that for some $c \in C$,

$$
\sum_{i=1}^{N} c_{i}\left(\frac{r_{i}+t_{i}}{2}, y_{i}, \widehat{z}_{i}\right)=\left(\sum_{i=1}^{N} c_{i}\left(\frac{r_{i}+t_{i}}{2}\right), y, \widehat{z}\right),
$$

where $y=\sum_{i} c_{i} y_{i}$ and $\widehat{z}=\sum_{i} c_{i} \widehat{z}_{i}$ Thus for $a \in\{r, t\}$, we have

$$
\sum_{i=1}^{N} c_{i}\left(a_{i}, y_{i}, \widehat{z}_{i}\right)=\left(\sum_{i=1}^{N} c_{i} a_{i}, y, \widehat{z}\right) \in \oplus_{C}\left(\left\{B_{\rho_{i}}^{s}\left(a_{i}, y_{i}\right)\right\}\right),
$$

hence $\ell_{a}(0, y, \widehat{z}) \leq \sum_{i=1}^{N} c_{i} a_{i} \leq u_{a}(0, y, \widehat{z})$. Thus,

$$
\ell(0, y, \widehat{z}) \leq \frac{1}{2} \sum_{i=1}^{N} c_{i} r_{i}+\frac{1}{2} \sum_{i=1}^{N} c_{i} t_{i} \leq u(0, y, \widehat{z}),
$$


which shows that $w \in E$ and establishes (4.6). Hence

$$
\begin{aligned}
|E| & =\int_{D}(u(v)-\ell(v)) d v \\
& =\frac{1}{2} \int_{D}\left(u_{r}-\ell_{r}\right)(v) d v+\frac{1}{2} \int_{D}\left(u_{t}-\ell_{t}\right)(v) d v \\
& =\frac{1}{2}\left|\oplus_{C}\left(\left\{B_{\rho_{i}}^{s}\left(r_{i}, y_{i}\right)\right\}\right)\right|+\frac{1}{2}\left|\oplus_{C}\left(\left\{B_{\rho_{i}}^{s}\left(t_{i}, y_{i}\right)\right\}\right)\right|,
\end{aligned}
$$

which completes the proof.

\section{MAIN PROOFS}

Proof of Theorem 1.2. For $\left(x_{1}, z_{1}\right), \ldots,\left(x_{N}, z_{N}\right) \in \mathbb{R}^{n} \times[0, \infty)$, write $w_{i}=$ $\left(x_{i}, z_{i}\right)$ and let $T_{\left\{w_{i}\right\}}$ be the least log-concave function supported on the set $\operatorname{conv}\left\{x_{1}, \ldots, x_{N}\right\}$ with $T\left(x_{i}\right) \geq z_{i}, i=1, \ldots, N$, i.e.,

$$
T_{\left\{w_{i}\right\}}(x)=\exp \left(\sup \left\{z:(x, z) \in \operatorname{conv}\left\{\left(x_{1}, \log z_{1}\right), \ldots,\left(x_{N}, \log z_{N}\right)\right\}\right) .\right.
$$

With this notation, we set

$$
F\left(w_{1}, \ldots, w_{N}\right)=\int_{\mathbb{R}^{n}} T_{\left\{w_{i}\right\}}(x) d x .
$$

Let $f: \mathbb{R}^{n} \rightarrow[0, \infty)$ be an integrable log-concave function. Sample independent random vectors $W_{i}=\left(X_{i}, Z_{i}\right), i=1, \ldots, N$, according to the uniform Lebesgue measure on $G_{f}\left(\mathrm{cf} .(\underline{1.8})\right.$ ). Then the random function $[f]_{N}$ defined as in (1.10) satisfies

$$
\begin{aligned}
\mathbb{P}\left(\int_{\mathbb{R}^{n}}[f]_{N}(x) d x>\alpha\right) & =\mathbb{E}_{\{F>\alpha\}}\left(W_{1}, \ldots, W_{N}\right) \\
& =\frac{1}{\|f\|_{1}^{N}} \int_{N} \mathbb{1}_{\{F>\alpha\}}(\bar{w}) \prod_{i=1}^{N} \mathbb{1}_{\left[0, f\left(x_{i}\right)\right]}\left(z_{i}\right) d \bar{w}
\end{aligned}
$$

where $\int_{N}$ denotes the integral on $\left(\mathbb{R}^{n} \times[0, \infty)\right)^{N}$ and

$$
\bar{w}=\left(w_{1}, \ldots, w_{N}\right), \quad d \bar{w}=d w_{1} \ldots d w_{N} .
$$

It is sufficient to prove the theorem for $f_{\varepsilon}(x):=f(x) \mathbb{1}_{\{f>\varepsilon\}}(x)$ for each fixed $\varepsilon>0$. To see this, note that $\left(f_{\varepsilon}\right)^{*}=\left(f^{*}\right)_{\varepsilon}$. Thus if $W_{i}^{\varepsilon}=\left(X_{i}^{\varepsilon}, Z_{i}^{\varepsilon}\right)$, $i=1, \ldots, N$ are independent random vectors distributed according to the 
uniform Lebesgue measure on $G_{f_{\varepsilon}}$, we have

$$
\begin{aligned}
\mathbb{P}\left(\int_{\mathbb{R}^{n}}\left[f_{\varepsilon}\right]_{N}(x) d x>\alpha\right) & =\mathbb{E} \mathbb{1}_{\{F>\alpha\}}\left(W_{1}^{\varepsilon}, \ldots, W_{N}^{\varepsilon}\right) \\
& =\frac{1}{\left\|f_{\epsilon}\right\|_{1}^{N}} \int_{N} \mathbb{1}_{\{F\}>\alpha\}}(\bar{w}) \prod_{i=1}^{N} \mathbb{1}_{\left[\varepsilon, f\left(x_{i}\right)\right]}\left(z_{i}\right) d \bar{w} .
\end{aligned}
$$

Since $\left\|f_{\varepsilon}\right\|_{1} \rightarrow\|f\|_{1}$ and $f_{\varepsilon} \leq f$, dominated convergence implies

$$
\mathbb{P}\left(\int_{\mathbb{R}^{n}}[f]_{N}(x) d x>\alpha\right)=\lim _{\epsilon \rightarrow 0} \mathbb{P}\left(\int_{\mathbb{R}^{n}}\left[f_{\varepsilon}\right]_{N}(x) d x>\alpha\right) .
$$

For $s>0$ and $x \in \mathbb{R}^{n}$, we define

$$
\left[f_{\epsilon}\right]_{N, s}(x)=\left(1+\frac{\log \left[f_{\epsilon}\right]_{N}(x)}{s}\right)_{+}^{s} .
$$

For every $x$, we have almost sure convergence $\left[f_{\varepsilon}\right]_{N, s}(x) \rightarrow\left[f_{\varepsilon}\right]_{N}(x)$ as $s \rightarrow \infty$. Since $\left[f_{\varepsilon}\right]_{N, s},\left[f_{\varepsilon}\right]_{N}$ are dominated by $f$, this implies almost sure convergence of the integrals

$$
\int_{\mathbb{R}^{n}}\left[f_{\varepsilon}\right]_{N}(x) d x=\lim _{s \rightarrow 0} \int_{\mathbb{R}^{n}}\left[f_{\varepsilon}\right]_{N, s}(x) d x .
$$

The latter implies that

$$
\mathbb{P}\left(\int_{\mathbb{R}^{n}}\left[f_{\varepsilon}\right]_{N}(x) d x \leq \alpha\right)=\lim _{s \rightarrow \infty} \mathbb{P}\left(\int_{\mathbb{R}^{n}}\left[f_{\varepsilon}\right]_{N, s}(x) d x \leq \alpha\right)
$$

at all continuity points of

$$
[0, \infty) \ni a \mapsto \mathbb{P}\left(\int_{\mathbb{R}^{n}}\left[f_{\varepsilon}\right]_{N}(x) d x \leq a\right) .
$$

However, since $N>n+1$ and $f_{\varepsilon}$ is absolutely continuous with respect to Lebesgue measure, the random variable $\int_{\mathbb{R}^{n}}\left[f_{\varepsilon}\right]_{N}(x) d x$ is positive almost surely and (5.5) is continuous on $[0, \infty)$.

Thus it is sufficient to prove the theorem for $\left[f_{\varepsilon}\right]_{N, s}$ with $s$ large enough. For $s>-\log \epsilon$, we have $R_{i}:=R_{i}\left(Z_{1}^{\varepsilon}\right):=\left(1+\frac{\log Z_{i}^{\varepsilon}}{s}\right)>0$ for all $i \in$ $\{1, \ldots, N\}$. Then

$$
\left[f_{\epsilon}\right]_{N, s}(x)=\sup \left\{z^{s} \in \mathbb{R}:(x, z) \in H_{f_{\epsilon}, N, s}\right\},
$$

where

$$
H_{f_{\epsilon}, N, s}:=\operatorname{conv}\left\{\left(X_{1}^{\varepsilon}, R_{1}\left(Z_{1}^{\varepsilon}\right)\right), \ldots,\left(X_{N}^{\varepsilon}, R_{N}\left(Z_{N}^{\varepsilon}\right)\right)\right\} .
$$

By Lemma 3.1,

$$
\mathcal{K}_{\left[f_{\epsilon}\right]_{N, s}}^{s}=\operatorname{conv}\left\{B_{R_{1}}^{s}\left(X_{1}^{\varepsilon}\right), \ldots, B_{R_{N}}^{s}\left(X_{N}^{\varepsilon}\right)\right\} .
$$


By (3.3),

$$
\int_{\mathbb{R}^{n}}\left[f_{\varepsilon}\right]_{N, s}(x) d x=\kappa_{s}^{-1}\left|\mathcal{K}_{\left[f_{\varepsilon}\right]_{N, s}}^{s}\right|
$$

For $z_{1}, \ldots, z_{N} \in[0, \infty)$, we write $\rho_{i}=\rho_{i}\left(z_{i}\right)=\left(1+\frac{\log z_{i}}{s}\right)$ and define

$$
F_{s}\left(\left(x_{1}, z_{1}\right), \ldots,\left(x_{N}, z_{N}\right)\right)=\left|\operatorname{conv}\left\{B_{\rho_{1}}^{s}\left(x_{1}\right), \ldots, B_{\rho_{N}}^{s}\left(x_{N}\right)\right\}\right| .
$$

Then for each fixed $z_{1}, \ldots, z_{N}$,

$$
\left(x_{1}, \ldots, x_{N}\right) \mapsto F_{s}\left(\left(x_{1}, z_{1}\right), \ldots,\left(x_{N}, z_{N}\right)\right)
$$

is a Steiner convex function by Proposition 4.2. Applying Fubini, writing $d \bar{w}=d \bar{x} d \bar{z}$ and invoking Theorem 4.1, we obtain

$$
\begin{aligned}
\mathbb{P}\left(\left|\mathcal{K}_{\left[f_{\varepsilon}\right]_{N, s}}^{s}\right|>\alpha\right) & =\frac{1}{\left\|f_{\varepsilon}\right\|_{1}^{N}} \int_{N} \mathbb{1}_{\left\{F_{s}>\alpha\right\}}(\bar{w}) \prod_{i=1}^{N} \mathbb{1}_{\left[\varepsilon, f\left(x_{i}\right)\right]}\left(z_{i}\right) d \bar{w} \\
& =\frac{1}{\left\|f_{\varepsilon}\right\|_{1}^{N}} \int_{[0, \infty)^{N}}\left(\int_{\left(\mathbb{R}^{n}\right)^{N}} \mathbb{1}_{\left\{F_{s}>\alpha\right\}} \prod_{i=1}^{N} \mathbb{1}_{\left[\varepsilon, f\left(x_{i}\right)\right]}\left(z_{i}\right) d \bar{x}\right) d \bar{z} \\
& \geq \frac{1}{\left\|f_{\varepsilon}^{*}\right\|_{1}^{N}} \int_{[0, \infty)^{N}}\left(\int_{\left(\mathbb{R}^{n}\right)^{N}} \mathbb{1}_{\left\{F_{s}>\alpha\right\}} \prod_{i=1}^{N} \mathbb{1}_{\left[\varepsilon, f^{*}\left(x_{i}\right)\right]}\left(z_{i}\right) d \bar{x}\right) d \bar{z} \\
& =\frac{1}{\left\|f_{\varepsilon}^{*}\right\|_{1}^{N}} \int_{N} \mathbb{1}_{\left\{F_{s}>\alpha\right\}}(\bar{w}) \prod_{i=1}^{N} \mathbb{1}_{\left[\varepsilon, f^{*}\left(x_{i}\right)\right]}\left(z_{i}\right) d \bar{w} \\
& =\mathbb{P}\left(\left|\mathcal{K}_{\left[f_{\varepsilon}^{*}\right]_{N, s}}\right|>\alpha\right) .
\end{aligned}
$$

The result now follows from (5.6) applied to $f_{\varepsilon}^{*}$.

Proof of Theorem 1.1. For $w_{i}=\left(x_{i}, z_{i}\right) \in \mathbb{R}^{n} \times \mathbb{R}^{s}, i=1, \ldots, M+N$, we set

$$
F\left(w_{1}, \ldots, w_{N+M}\right)=\int_{\mathbb{R}^{n}} T_{\left\{w_{i}\right\}}^{(N)} \star_{\lambda} T_{\left\{w_{i}\right\}}^{(M)}(v) d v,
$$

where $T_{\left\{w_{i}\right\}}^{(N)}$ and $T_{\left\{w_{i}\right\}}^{(M)}$ are the least log-concave functions above the collections $\left\{w_{i}\right\}_{i \leq N}$ and $\left\{w_{i}\right\}_{N+1 \leq i \leq M}$, respectively (as defined as in (5.1)). Then the stochastic approximations $[f]_{N},[g]_{M}$ to $f, g$ satisfy

$$
\begin{aligned}
& \mathbb{P}\left(\int_{\mathbb{R}^{n}}[f]_{N} \star_{\lambda}[g]_{M}(v) d v>\alpha\right) \\
& \quad=\frac{1}{\prod_{i=1}^{M+N}\left\|h_{i}\right\|_{1}} \int_{N+M} \mathbb{1}_{\{F>\alpha\}}(\bar{w}) \prod_{i=1}^{N+M} \mathbb{1}_{\left[0, h_{i}\left(x_{i}\right)\right]}\left(z_{i}\right) d \bar{w}
\end{aligned}
$$


where $\int_{N+M}$ is the integral on $\left(\mathbb{R}^{n} \times[0, \infty)\right)^{N+M}, \bar{w}$ and $d \bar{w}$ are as in (5.2), $h_{i}=f_{i}$ for $i=1, \ldots, N$ and $h_{i}=g_{i}$ for $i=N+1, \ldots, N+M$. For $\varepsilon>0$, we apply the latter identity with $f_{\varepsilon}$ and $g_{\varepsilon}$ and use dominated convergence to get

$$
\mathbb{P}\left(\int_{\mathbb{R}^{n}}[f]_{N} \star_{\lambda}[g]_{M}(v) d v>\alpha\right)=\lim _{\varepsilon \rightarrow 0} \mathbb{P}\left(\int_{\mathbb{R}^{n}}\left[f_{\epsilon}\right]_{N} \star_{\lambda}\left[g_{\epsilon}\right]_{M}(v) d v>\alpha\right) .
$$

For $\varepsilon>0$, we sample independent random vectors $\left\{\left(X_{i}^{\varepsilon}, Z_{i}^{\varepsilon}\right)\right\}_{i=1}^{N}$ uniformly in $G_{f_{\varepsilon}}$ and $\left\{\left(X_{i}^{\varepsilon}, Z_{i}^{\varepsilon}\right)\right\}_{i=N+1}^{N+M}$ uniformly in $G_{g_{\varepsilon}}$. For $s>-\log \varepsilon$, we define $\left[f_{\epsilon}\right]_{N, s}$, and $\left[g_{\epsilon}\right]_{M, s}$ as in (5.3). Note that for each $v \in \mathbb{R}^{n}$, we have almost sure convergence

$$
\left(\left[f_{\epsilon}\right]_{N} \star_{\lambda}\left[g_{\epsilon}\right]_{M}\right)(v)=\lim _{s \rightarrow \infty}\left(\left[f_{\epsilon}\right]_{N, s} \star_{\lambda, s}\left[g_{\epsilon}\right]_{M, s}\right)(v) .
$$

Since $\left[f_{\epsilon}\right]_{N, s} \star_{\lambda, s}\left[g_{\epsilon}\right]_{M, s}$ and $\left[f_{\epsilon}\right]_{N} \star_{\lambda}\left[g_{\epsilon}\right]_{M}$ are dominated by $f \star_{\lambda} g$, we can argue as in the proof of Theorem 1.2 to get, for each $\alpha \geq 0$,

$$
\begin{aligned}
& \mathbb{P}\left(\int_{\mathbb{R}^{n}}\left(\left[f_{\epsilon}\right]_{N} \star_{\lambda}\left[g_{\epsilon}\right]_{M}\right)(v) d v \leq \alpha\right) \\
& \quad=\lim _{s \rightarrow \infty} \mathbb{P}\left(\int_{\mathbb{R}^{n}}\left(\left[f_{\epsilon}\right]_{N, s} \star_{\lambda, s}\left[g_{\epsilon}\right]_{M, s}\right)(v) d v \leq \alpha\right) .
\end{aligned}
$$

Since $\left[f_{\epsilon}\right]_{N, s} \star_{\lambda, s}\left[g_{\epsilon}\right]_{M, s}$ is $s$-concave, the body $\mathcal{K}_{\left[f_{\epsilon}\right]_{N, s} \star_{\lambda, s}\left[g_{\epsilon}\right]_{M, s}}$ is convex. Moreover, by (3.3),

$$
\int_{\mathbb{R}^{n}}\left(\left[f_{\epsilon}\right]_{N, s} \star_{\lambda, s}\left[g_{\epsilon}\right]_{M, s}\right)(v) d v=\kappa_{s}^{-1}\left|K_{\left[f_{\epsilon}\right]_{N, s} \star_{\lambda, s}\left[g_{\epsilon}\right]_{M, s}}\right| .
$$

Recalling (3.4), we have

$$
\begin{aligned}
\mathcal{K}_{\left[f_{\epsilon}\right]_{N, s} \star_{\lambda, s}\left[g_{\epsilon}\right]_{M, s}}^{s} & =\lambda \mathcal{K}_{\left[f_{\epsilon}\right]_{N, s}}^{s}+(1-\lambda) \mathcal{K}_{\left[g_{\varepsilon}\right]_{M, s}}^{s} \\
& =\mathcal{K}_{\left[f_{\epsilon}\right]_{N, s}}^{s}+{ }_{\lambda} \mathcal{K}_{\left[g_{\varepsilon}\right]_{M, s}}^{s} .
\end{aligned}
$$

Set $R_{i}:=R_{i}\left(Z_{i}\right):=\left(1+\frac{\log Z_{i}^{\varepsilon}}{s}\right)$. By Lemma 3.1, we have

$$
\begin{aligned}
\mathcal{K}_{\left[f_{\epsilon}\right]_{N, s}}^{s} & =\operatorname{conv}\left\{B_{R_{1}}^{s}\left(X_{1}\right), \ldots, B_{R_{N}}^{s}\left(X_{N}\right)\right\} \\
& =\oplus_{C_{N}}\left(\left\{B_{R_{i}}^{s}\left(X_{i}\right)\right\}_{i=1}^{N}\right),
\end{aligned}
$$

where $C_{N}=\operatorname{conv}\left\{e_{1}, \ldots, e_{N}\right\}$. Similarly,

$$
\begin{aligned}
\mathcal{K}_{\left[g_{\epsilon}\right]_{M, s}}^{s} & =\operatorname{conv}\left\{B_{R_{N+1}}^{s}\left(X_{N+1}\right), \ldots, B_{R_{N+M}}^{s}\left(X_{N+M}\right)\right\} \\
& =\oplus_{C_{M}}\left(\left\{B_{R_{i}}^{s}\left(X_{i}\right)\right\}_{i=N+1}^{M}\right),
\end{aligned}
$$


where $C_{M}=\operatorname{conv}\left\{e_{1}, \ldots, e_{M}\right\}$. Thus if we write $\widehat{C}_{M}=\operatorname{conv}\left\{e_{N+1}, \ldots, e_{N+M}\right\}$, (which is similar to (4.4)), we have

$$
\begin{aligned}
\mathcal{K}_{\left[f_{\epsilon}\right]_{N, s} \star_{\lambda, s}\left[g_{\epsilon}\right]_{M, s}}^{s} & =\oplus_{C_{N}}\left(\left\{B_{R_{i}}^{s}\left(X_{i}\right)\right\}_{i=1}^{N}\right)+_{\lambda} \oplus_{C_{M}}\left(\left\{B_{R_{i}}^{s}\left(X_{i}\right)\right\}_{i=N+1}^{N+M}\right) \\
& =\oplus_{C_{N}+{ }_{\lambda} \widehat{C}_{M}}\left(\left\{B_{R_{i}}^{s}\left(X_{i}\right)\right\}_{i=1}^{N+M}\right) .
\end{aligned}
$$

For $z_{1}, \ldots, z_{N+M} \in[0, \infty)$, write $\rho_{i}=\rho_{i}\left(z_{i}\right)=\left(1+\frac{\log z_{i}}{s}\right)$ and define

$$
F_{s}\left(\left(x_{1}, z_{1}\right), \ldots,\left(x_{N+M}, z_{N+M}\right)\right)=\left|\oplus_{C_{N}+\lambda \widehat{C}_{M}}\left(\left\{B_{\rho_{i}}^{s}\left(x_{i}\right)\right\}_{i=1}^{N+M}\right)\right| .
$$

Then for each fixed $z_{1}, \ldots, z_{N+M}$, the function

$$
\left(x_{1}, \ldots, x_{N+M}\right) \mapsto F_{s}\left(\left(x_{1}, z_{1}\right), \ldots,\left(x_{N+M}, z_{N+M}\right)\right)
$$

is Steiner convex by Proposition 4.2. By Fubini and Theorem 4.1, we have

$$
\begin{aligned}
& \mathbb{P}\left(\mid \mathcal{K}_{\left[f_{\varepsilon}\right]_{N, s^{\star} \lambda, s}\left[g_{\varepsilon}\right]_{N, s}}^{s}>\alpha\right) \\
& \quad=\frac{1}{\prod_{i=1}^{N+M}\left\|\left(h_{i}\right)_{\varepsilon}\right\|_{1}} \int_{N+M} \mathbb{1}_{\left\{F_{s}>\alpha\right\}}(\bar{w}) \prod_{i=1}^{N+M} \mathbb{1}_{\left[\varepsilon, h_{i}\left(x_{i}\right)\right]}\left(z_{i}\right) d \bar{w} \\
& =\frac{1}{\prod_{i=1}^{N+M}\left\|\left(h_{i}\right)_{\varepsilon}\right\|_{1}} \int_{[0, \infty)^{N+M}}\left(\int_{\left(\mathbb{R}^{n}\right)^{N+M}} \mathbb{1}_{\left\{F_{s}>\alpha\right\}}(\bar{w}) \prod_{i=1}^{N+M} \mathbb{1}_{\left[\varepsilon, h_{i}\left(x_{i}\right)\right]}\left(z_{i}\right) d \bar{x}\right) d \bar{z} \\
& \geq \frac{1}{\prod_{i=1}^{N+M}\left\|\left(h_{i}\right)_{\varepsilon}^{*}\right\|_{1}} \int_{[0, \infty)^{N+M}}\left(\int_{\left(\mathbb{R}^{n}\right)^{N+M}} \mathbb{1}_{\left\{F_{s}>\alpha\right\}}(\bar{w}) \prod_{i=1}^{N+M} \mathbb{1}_{\left[\varepsilon, h_{i}^{*}\left(x_{i}\right)\right]}\left(z_{i}\right) d \bar{x}\right) d \bar{z} \\
& \quad=\frac{1}{\prod_{i=1}^{N+M}\left\|\left(h_{i}\right)_{\varepsilon}^{*}\right\|_{1}} \int_{N+M} \mathbb{1}_{\left\{F_{s}>\alpha\right\}}(\bar{w}) \prod_{i=1}^{N+M} \mathbb{1}_{\left[\varepsilon, h_{i}^{*}\left(x_{i}\right)\right]}\left(z_{i}\right) d \bar{w} \\
& \quad=\mathbb{P}\left(\left|\mathcal{K}_{\left[f_{\varepsilon}^{*}\right]_{N, s} s_{\lambda, s}\left[g_{\varepsilon}^{*}\right]_{N, s}}\right|>\alpha\right) .
\end{aligned}
$$

The result now follows from (5.7) applied to $f_{\varepsilon}^{*}$ and $g_{\varepsilon}^{*}$.

Acknowledgments It is our pleasure to thank Dario Cordero-Erausquin, Matthieu Fradelizi, Mokshay Madiman and Grigoris Paouris for helpful correspondence, discussions and feedback. P. Pivovarov was supported by NSF grant DMS-1612936 and Simons Foundation grant \#635531.

\section{REFERENCES}

[1] S. Artstein-Avidan, D.I. Florentin, and A. Segal. Polar Prékopa-Leindler inequalities. Preprint. https://arxiv.org/abs/1707.08732. 
[2] S. Artstein-Avidan, B. Klartag, and V. Milman. The Santaló point of a function, and a functional form of the Santaló inequality. Mathematika, 51(1-2):33-48 (2005), 2004.

[3] S. Artstein-Avidan, B. Klartag, C. Schütt, and E. Werner. Functional affineisoperimetry and an inverse logarithmic Sobolev inequality. J. Funct. Anal., 262(9):4181-4204, 2012.

[4] K. Ball. Isoperimetric problems in $\ell_{p}$ and sections of convex sets. $\mathrm{PhD}$ thesis, Cambridge, 1986.

[5] F. Barthe and M. Fradelizi. The volume product of convex bodies with many hyperplane symmetries. Amer. J. Math., 135(2):311-347, 2013.

[6] W. Blaschke. Über affine geometrie xi: Lösung des "vierpunktproblems" von Sylvester aus der theorie der geometrischen wahrscheinlichkeiten. Leipziger Berichte, 69:436-453, 1917.

[7] S. G. Bobkov, A. Colesanti, and I. Fragalà. Quermassintegrals of quasi-concave functions and generalized Prékopa-Leindler inequalities. Manuscripta Math., 143(12):131-169, 2014.

[8] C. Borell. Convex set functions in $d$-space. Period. Math. Hungar., 6(2):111-136, 1975.

[9] H. J. Brascamp and E. H. Lieb. Best constants in Young's inequality, its converse, and its generalization to more than three functions. Advances in Math., 20(2):151$173,1976$.

[10] H. J. Brascamp and E. H. Lieb. On extensions of the Brunn-Minkowski and PrékopaLeindler theorems, including inequalities for log concave functions, and with an application to the diffusion equation. J. Funct. Anal., 22(4):366-389, 1976.

[11] H. J. Brascamp, E. H. Lieb, and J. M. Luttinger. A general rearrangement inequality for multiple integrals. J. Functional Analysis, 17:227-237, 1974.

[12] A. Burchard. A short course on rearrangement inequalities. Avaible at http://www.math.utoronto.ca/almut/rearrange/pdf.

[13] H. Busemann. Volume in terms of concurrent cross-sections. Pacific J. Math., 3:1-12, 1953.

[14] U. Caglar, M. Fradelizi, O. Guédon, J. Lehec, C. Schütt, and E. M. Werner. Functional versions of $L_{p}$-affine surface area and entropy inequalities. Int. Math. Res. Not. IMRN, 2016(4):1223-1250, 2016.

[15] S. Campi, A. Colesanti, and P. Gronchi. A note on Sylvester's problem for random polytopes in a convex body. Rend. Istit. Mat. Univ. Trieste, 31(1-2):79-94, 1999.

[16] M. Christ. Estimates for the $k$-plane transform. Indiana Univ. Math. J., 33(6):891910, 1984.

[17] D. Cordero-Erausquin. On matrix-valued log-concavity and related Prékopa and Brascamp-Lieb inequalities. Adv. Math., 351:96-116, 2019.

[18] D. Cordero-Erausquin and B. Klartag. Interpolations, convexity and geometric inequalities. In Geometric aspects of functional analysis, volume 2050 of Lecture Notes in Math., pages 151-168. Springer, Heidelberg, 2012.

[19] D. Cordero-Erausquin and B. Maurey. Some extensions of the Prékopa-Leindler inequality using Borell's stochastic approach. Studia Math., 238(3):201-233, 2017.

[20] D. Cordero-Erausquin, R. J. McCann, and M. Schmuckenschläger. A Riemannian interpolation inequality à la Borell, Brascamp and Lieb. Invent. Math., 146(2):219257, 2001. 
[21] M. Fradelizi and M. Meyer. Some functional forms of Blaschke-Santaló inequality. Math. Z., 256(2):379-395, 2007.

[22] M. Fradelizi and M. Meyer. Some functional inverse Santaló inequalities. Adv. Math., 218(5):1430-1452, 2008.

[23] R. J. Gardner. The Brunn-Minkowski inequality. Bull. Amer. Math. Soc. (N.S.), 39(3):355-405, 2002.

[24] R. J. Gardner, D. Hug, and W. Weil. The Orlicz-Brunn-Minkowski theory: a general framework, additions, and inequalities. J. Differential Geom., 97(3):427-476, 2014.

[25] R.J. Gardner, D. Hug, and W. Weil. Operations between sets in geometry. J. Eur. Math. Soc. (JEMS), 15(6):2297-2352, 2013.

[26] A. Giannopoulos and A. Tsolomitis. Volume radius of a random polytope in a convex body. Math. Proc. Cambridge Philos. Soc., 134(1):13-21, 2003.

[27] H. Groemer. On the mean value of the volume of a random polytope in a convex set. Arch. Math. (Basel), 25:86-90, 1974.

[28] B. Klartag. Marginals of geometric inequalities. In Geometric aspects of functional analysis, volume 1910 of Lecture Notes in Math., pages 133-166. Springer, Berlin, 2007.

[29] B. Klartag and V. D. Milman. Geometry of log-concave functions and measures. Geom. Dedicata, 112:169-182, 2005.

[30] J. Lehec. A direct proof of the functional Santaló inequality. C. R. Math. Acad. Sci. Paris, 347(1-2):55-58, 2009.

[31] L. Leindler. On a certain converse of Hölder's inequality. II. Acta Sci. Math. (Szeged), 33(3-4):217-223, 1972.

[32] E. H. Lieb and M. Loss. Analysis, volume 14 of Graduate Studies in Mathematics. American Mathematical Society, Providence, RI, second edition, 2001.

[33] E. Lutwak, D. Yang, and G. Zhang. Orlicz centroid bodies. J. Differential Geom., 84(2):365-387, 2010.

[34] M. Madiman, J. Melbourne, and P. Xu. Forward and reverse entropy power inequalities in convex geometry. In Convexity and concentration, volume 161 of IMA Vol. Math. Appl., pages 391-425. Springer, New York, 2017.

[35] J. Melbourne. Rearrangement and prékopa-leindler type inequalities. Avaible at https://arxiv.org/abs/1806.08837.

[36] V. Milman and L. Rotem. Mixed integrals and related inequalities. J. Funct. Anal., 264(2):570-604, 2013.

[37] G. Paouris and P. Pivovarov. A probabilistic take on isoperimetric-type inequalities. Adv. Math., 230(3):1402-1422, 2012.

[38] G. Paouris and P. Pivovarov. Randomized isoperimetric inequalities. In Convexity and concentration, volume 161 of IMA Vol. Math. Appl., pages 391-425. Springer, New York, 2017.

[39] R. E. Pfiefer. The extrema of geometric mean values. ProQuest LLC, Ann Arbor, MI, 1982. Thesis (Ph.D.)-University of California, Davis.

[40] A. Prékopa. Logarithmic concave measures with application to stochastic programming. Acta Sci. Math. (Szeged), 32:301-316, 1971.

[41] A. Prékopa. On logarithmic concave measures and functions. Acta Sci. Math. (Szeged), 34:335-343, 1973.

[42] Y. Rinott. On convexity of measures. Ann. Probability, 4(6):1020-1026, 1976.

[43] C. A. Rogers. A single integral inequality. J. London Math. Soc., 32:102-108, 1957. 
[44] C. A. Rogers and G. C. Shephard. Some extremal problems for convex bodies. Mathematika, 5:93-102, 1958.

[45] R. Schneider. Convex bodies: the Brunn-Minkowski theory, volume 151 of Encyclopedia of Mathematics and its Applications. Cambridge University Press, Cambridge, expanded edition, 2014.

[46] B. Simon. Convexity, volume 187 of Cambridge Tracts in Mathematics. Cambridge University Press, Cambridge, 2011. An analytic viewpoint.

University of Missouri, Department of Mathematics, Columbia MO 65201

E-mail address: pivovarovp@missouri.edu \& jrc65@mail.missouri.edu 